\title{
Observation of Cosmic Ray Anisotropy with Nine Years of IceCube Data
}

\section{The IceCube Collaboration}

(a complete list of authors can be found at the end of the proceedings)

E-mail: mcnally_ft@mercer.edu

The IceCube Observatory has collected over 577 billion cosmic-ray induced muon events in its final configuration from May 2011 to May 2020. We used this data set to provide an unprecedented statistically accurate map of the cosmic ray arrival direction distribution in the TeV-PeV energy range scale in the Southern Hemisphere. Such an increase in event statistics makes it possible to extend the sensitivity to anisotropies at higher cosmic ray energies and smaller angular scales. It will also facilitate a more detailed assessment of the observatory stability over both short- and long-time scales. This will enable us to study the time variability of the cosmic ray anisotropy on a yearly-base and over the entire data sample period covering most of the solar cycle 24 . We present the preliminary results from the study with the extended event sample.

Corresponding authors: Frank McNally ${ }^{1 *}$, Rasha Abbasi ${ }^{2}$, Paolo Desiati ${ }^{3}$, Juan Carlos Díaz Vélez $^{3}$, Timothy Aguado ${ }^{2}$, Katherine Gruchot ${ }^{2}$, Andrew Moy $^{2}$, Alexander Simmons ${ }^{1}$, Andrew Thorpe $^{1}$, and Hannah Woodward ${ }^{4}$

${ }^{1}$ Mercer University, U.S.A.

${ }^{2}$ Loyola University Chicago, U.S.A.

${ }^{3}$ University of Wisconsin - Madison, U.S.A.

${ }^{4}$ University of Virginia, U.S.A. (UW-Madison REU student 2020)

* Presenter 


\section{Introduction}

With the recent discovery of galactic gamma-ray Pevatrons $[1,2]$ we are getting closer to the identification of the cosmic ray sources contributing to observations up to the knee. Traditionally, the measurement of cosmic ray energy and mass composition has been utilized by astrophysicists to infer global properties about their sources and their long propagation journey across the galactic magnetized medium. Now that their sources may have been identified, it is possible to improve our knowledge on how cosmic rays diffuse in the interstellar medium. Anisotropy - a property long observed in the arrival directions of cosmic rays - may provide additional hints linking cosmic rays to their sources of acceleration and, more likely, to their propagation through the inhomogeneous interstellar medium. On the one hand, ultra-high energy cosmic rays, whose direction is affected very little by astrophysical magnetized plasmas, can be used to identify their faraway extra-galactic sources [3, 4]. On the other hand, the arrival direction distribution of lower energy cosmic rays, of galactic origin, may be used to unfold the properties of the interstellar medium through which they propagated [5].

Ground-based experiments have observed the cosmic rays' anisotropy since the seventies, but in the last two decades a new generation of observatories has provided unprecedented and detailed observations over a wide energy range (TeV-PeV) [6-33]. The anisotropy is small (of order of $10^{-3}$ in relative intensity) and it changes with energy as if a transition between two distinct causes occurs around $100 \mathrm{TeV}$. Observations also show that the anisotropy has a complex angular structure, spanning from the large-scale dipole and quadrupole (thought to be associated with scattering off magnetic turbulence leading to pitch angle diffusion [34]) down to smaller scales with relative amplitude smaller than $10^{-4}$ (thought to be linked to non-diffusive processes within a scattering mean path [35, 36]). While new, large, ground-based experiments under construction (LHAASO [37]) and under design (SWGO [38, 39], IceCube-Gen2 [40]) will provide a leap in our anisotropy observations, existing experiments are still improving the quality of their data and analyses.

This work presents an update of cosmic ray anisotropy measurements in the energy range of $10 \mathrm{TeV}$ to a few PeV, observed by the IceCube Observatory using the 577 billion events collected from 2011 to 2020. The unprecedented stability of the data sample, acquired by using the same experimental configuration over time and by improving analysis techniques, makes it possible for the first time to probe into time variabilities of the anisotropy with minimal systematic uncertainties.

\section{Data Analysis}

This work is designed to serve as an update to previous anisotropy studies completed in IceCube [26-28, 30, 31]. We will therefore first highlight changes and improvements unique to this study before providing a brief overview of the overall method.

The dataset for this work consists of 577 billion cosmic ray events, collected over nine years by the in-ice component of IceCube. In addition to being $>80 \%$ larger than the previous sixyear publication [30], these events were all collected in the completed 86-string configuration, allowing for unprecedented statistics and stability in systematic checks. The 9-year time frame brings the observation period much closer to covering an 11-year solar cycle. A consistent detector 
configuration also means we can use a single simulation dataset in the creation and study of energydependent skymaps. In this study, we use improved simulations featuring post-LHC CORSIKA SIBYLL $2.3 \mathrm{c}$ and a factor of 10 increase in statistics. The simulations include an improved description of the detector and of the optical properties of the instrumented ice. The Monte Carlo data is weighted to a Gaisser-H4a [41, 42] composition model.

Aside from the aforementioned improvements, the analysis method was largely kept consistent with previous analyses [26-32] to allow for direct comparison of the results. The maps that follow rely on the comparison of detector data to a background map that simulates the detector response to an isotropic sky. The background map itself is not isotropic, but must account for real-world factors, including detector response as a function of atmospheric depth, and preferred azimuthal acceptances due to detector configuration. To create the background maps, we use the time-scrambling method detailed in [27]. In short, we store the arrival times of all events within a time-scrambling window. Then, for each event, we create 20 background events, each with a weight of $1 / 20$ and a time randomly selected from the time-

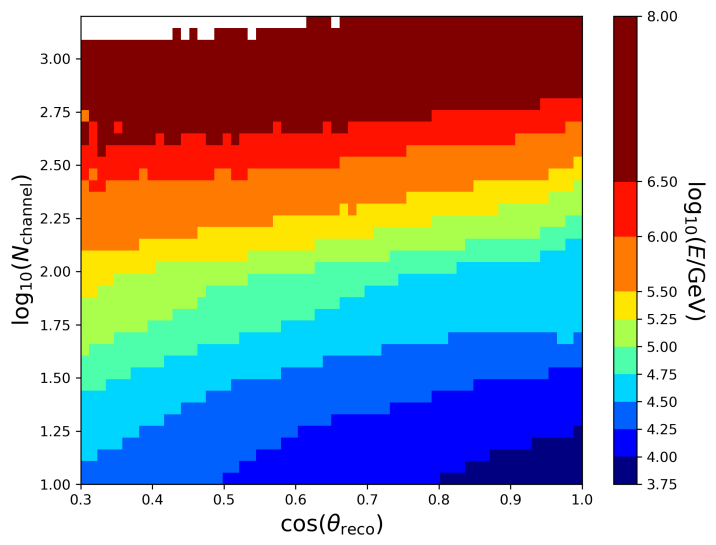

Figure 1: Median true energy of cosmic ray primary particles (from simulation) as a function of reconstructed zenith $\left(\theta_{\text {reco }}\right)$ and the number of DOMs triggered $\left(N_{\text {channel }}\right)$. scrambling window. By using local arrival coordinates and actual event times, the background map reflects the local angular and temporal event distribution seen by the detector. However, by choosing times from within a 24-hour timescrambling window, the sidereal locations of the background events are randomly distributed in right ascension, producing a uniform distribution folded with the detector response.

All maps are created using HEALPix ${ }^{1}$ software, which bins the sky into equal area bins aligned along declination bands. Setting the parameter $N_{\text {side }}=64$ produces bins with an angular size of $\left(0.84^{\circ}\right)^{2}$. The relative intensity $\delta I$ is then calculated as a fractional deviation of the data $N$ from background $\langle N\rangle$, using $\delta I=(N-\langle N\rangle) /\langle N\rangle$ on a pixel-by-pixel basis. Significance values are pre-trial, and calculated according to Li \& Ma [43]. The maps are smoothed by assigning to each pixel the combined value of all pixels within a given angular radius, a process known as "tophat" smoothing. For maps created from the full dataset, a $5^{\circ}$ smoothing radius is used, roughly corresponding to the $\ell$-value at which the power spectrum for IceCube is consistent with noise. For maps split by energy, a $20^{\circ}$ smoothing radius is used. This value was previously selected in order to maximize statistical significance while preserving our ability to observe large-scale structural changes.

To split the events into energy bins, we consider two parameters correlated with primary energy: the number of Digital Optical Modules (DOMs) hit ( $\left.N_{\text {channel }}\right)$, and the reconstructed zenith

\footnotetext{
${ }^{1}$ https://healpix.jpl.nasa.gov
} 
angle $\left(\theta_{\text {reco }}\right)$. While $N_{\text {channel }}$ is a simple counter that does not take into account track location or detector geometry, it should still, on average, increase with primary energy. The zenith-dependence of primary energy arises from the atmospheric depth the shower must penetrate before reaching the detector; lower-energy showers are filtered out at zenith angles closer to the horizon, their mean particle energy dropping below the detection threshold before the shower reaches the detector. It should be noted that, because IceCube is only sensitive to the muonic component of air showers, there are inherent limits on the energy resolution. As a result, while the energy bins are statistically independent, the energy distributions of adjacent energy bins have significant overlap. Using simulation, all events were binned in $\log _{10}\left(N_{\text {channel }}\right)$ and $\cos \left(\theta_{\text {reco }}\right)$. Figure 1 shows the resultant median energy value for each bin. After smoothing this table using splines (see method in [44]), the $N_{\text {channel }}$ and $\theta_{\text {reco }}$ values of each event were used to place it in an energy bin.

\section{Results}

A study of the sidereal anisotropy using all events is shown in Fig. 2. These maps are consistent with previous studies - at both large and small angular scales, we see the same features at the same locations. This study does yield higher pre-trial significance values, as expected given the increased sample size.
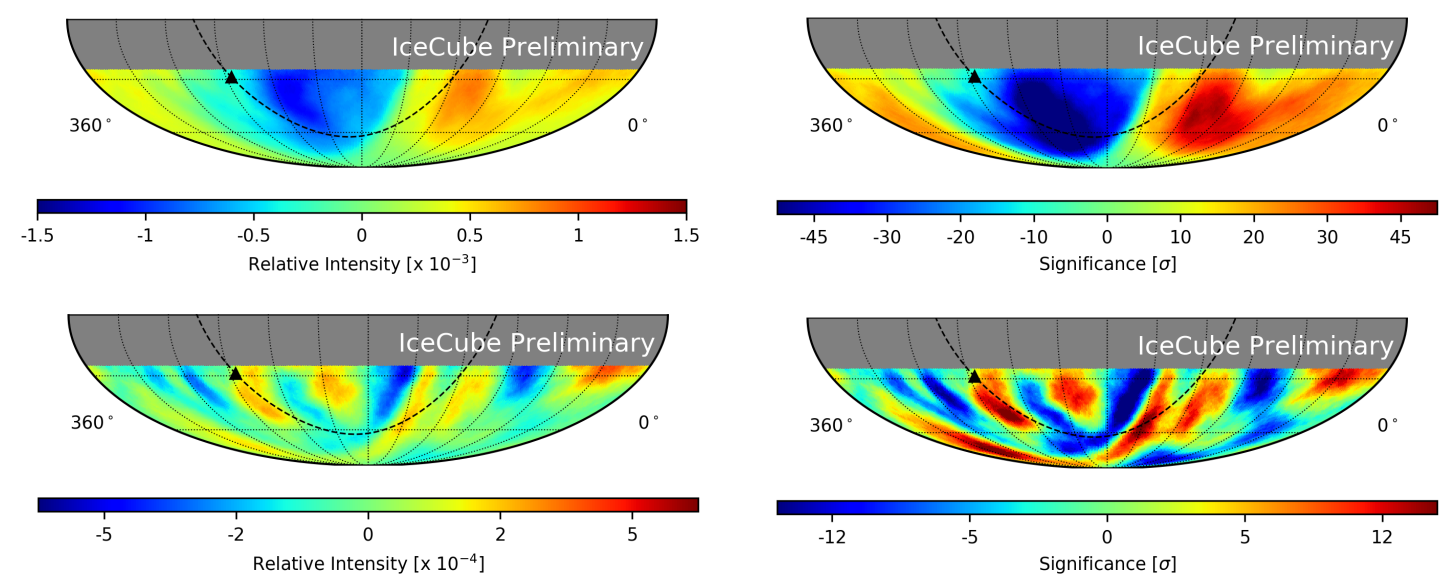

Figure 2: Relative intensity (left) and significance (right) maps, shown in equatorial coordinates for all data (top) and with best-fit dipole and quadrupole terms subtracted (bottom). The dashed line indicates the galactic plane, the triangle marks the galactic center.

To better visualize any potential time-dependence of the anisotropy, we can look at a onedimensional projection of the data along right ascension. To produce Fig. 3, the data was split by calendar year, from May $15^{\text {th }}$ of the listed year to May $14^{\text {th }}$ of the following. The shift from detector to calendar years provides a better grasp on our systematic uncertainties. The length of detector years is somewhat arbitrary, with some being much longer than others. When a time period significantly greater or less than 365 days is considered, the Compton-Getting effect due to the Earth's motion around the Sun does not cancel out. In the frequency domain, we are concerned with the contamination of the 366-day sidereal year by sidebands from the 365-day solar year. To determine the magnitude of this effect, we consider a 364-day "anti-sidereal" year [11]. By shifting 
to calendar years, the influence of any solar signal on the sidereal frame is minimized, visible as a diminished signal in the anti-sidereal frame. As a result, the systematic error bars, which once were much larger than the statistical errors, are now effectively equivalent. With the newly reduced systematic uncertainties and extended observation time, there appear to be time-related shifts in several of the right ascension bins. Studies are currently underway to determine the significance of the apparent effect, incorporating a recently-collected tenth year of data.

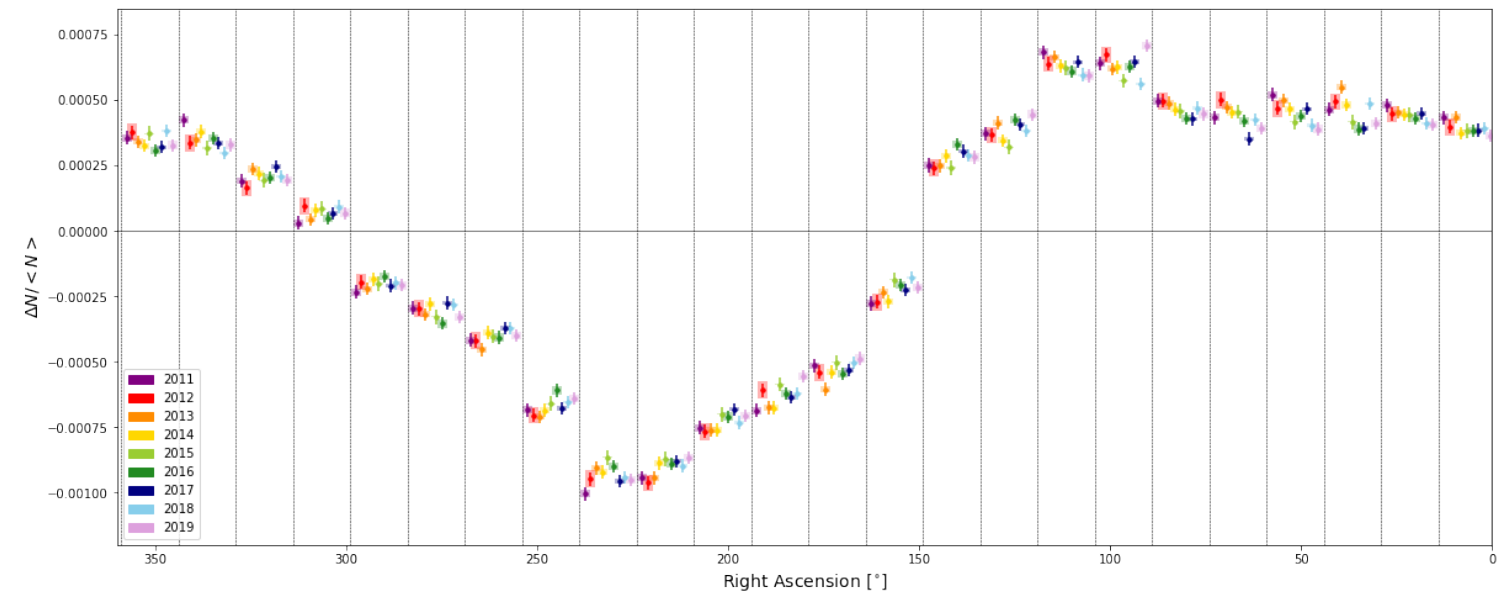

Figure 3: A one-dimensional projection of relative intensity binned in right ascension, split by detector year. The small, shaded regions around each point represent systematic uncertainties, calculated using the amplitude of the best-fit dipole to the anti-sidereal distribution for each year. The solid error bars are statistical.

The new simulation yields energy-dependent maps that are also consistent with previous results, as shown in Fig. 4. Large regions of excess and deficit $\left(+90^{\circ}\right.$ and $+270^{\circ}$, respectively) fade in strength with increasing energy, replaced by a dominant deficit near a right ascension of $+90^{\circ}$ around $100 \mathrm{TeV}$. While the locations and relative intensities of these structures are consistent with previous studies, the increased sample size pushes several of the features in the highest-energy map to $>3 \sigma$ (see Fig. 4). The significance of the features present in the 1-3 PeV map also changes; studies are underway to determine whether this is a time-dependent result, or an effect of the new simulation used to create our energy bins.

\section{Conclusions}

We present several improvements to previous IceCube analyses of the observed anisotropy in cosmic ray arrival direction. Nine years of data collection with the 86-string configuration of the in-ice component of IceCube provide an unprecedented sample size of 577 billion events. We correspondingly see large- and small-scale structures in the all-event sample at higher significance, with the morphology of these maps largely unchanged. A study of the energy-dependence yields a similar result; while the observed structures and transitions remain consistent, we can report features in our highest-energy map ( $>3 \mathrm{PeV}$ ) at a pre-trial significance of over $3 \sigma$. Changes to the energy maps are a function of both enhanced statistics and improvements to event simulation. 

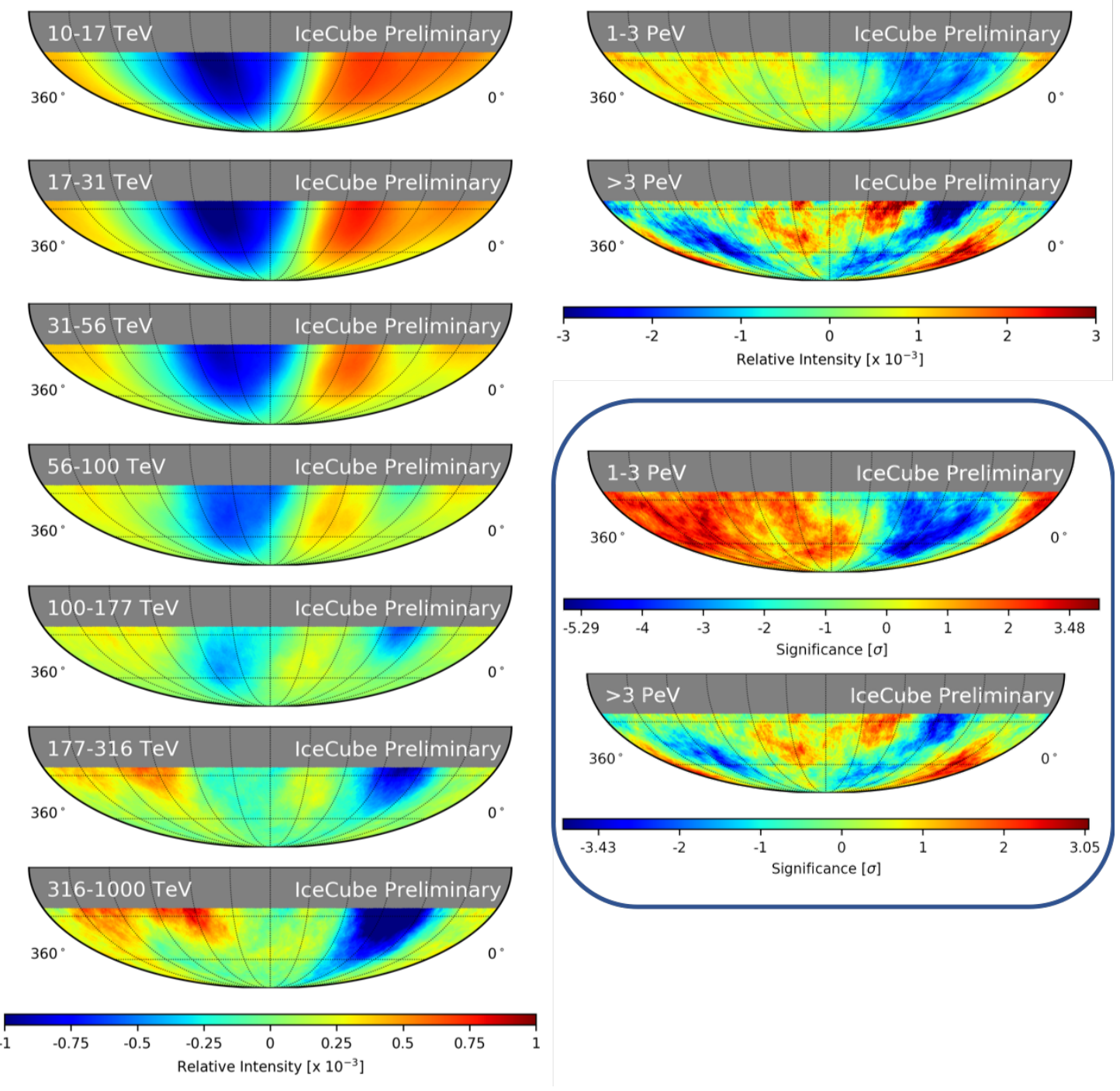

Figure 4: Relative intensity maps for each energy bin (left, top right) featuring top-hat smoothing with a $20^{\circ}$ angular radius. Both the features present and the change in structure are consistent with previous work. The scale is changed at $1 \mathrm{PeV}$ to avoid saturation. Also shown are significance maps for the two highest energy bins (bottom right).

Use of a consistent detector configuration also enables the study of data by calendar year as opposed to detector year. Systematic uncertainties in the sidereal anisotropy arise from interference with the solar frame, an effect that should largely cancel over a calendar year. As a result, our systematic uncertainties in the time-dependent study of the sidereal anisotropy (Fig. 3) are significantly reduced. As IceCube approaches 11 years of data collection in its 86-string configuration, this work should enhance our ability to search for any effect of the 11-year solar cycle on the observed cosmic ray anisotropy. 


\section{References}

[1] Tibet AS $\gamma$ Collaboration, (2021), Nature Astronomy 5, 460

[2] LHAASO Collaboration, Cao, Z. et al., (2021), Nature 594, 33

[3] Pierre Auger Collaboration, Aab, A. et al., (2017), Science 357, 1266

[4] TA Collaboration, Abbasi, R.U. et al., (2018) Astrophys. J. Lett., 867, L27

[5] Desiati, P., et al., (2021), Snowmass 2021 Letter of Intent, arXiv:2009.04883

[6] Nagashima, et al. (1998), J. of Geophys. Res. 1031, 17429

[7] Hall, D.L. et al., (1999), J. of Geophys. Res. 104, 6737

[8] Tibet AS $\gamma$ Collaboration, Amenomori, M. et al., (2005), Astrophys. J. Lett. 626, L29

[9] Tibet AS $\gamma$ Collaboration, Amenomori, M. et al., (2006), Science, 314, 439

[10] Tibet AS $\gamma$ Collaboration, Amenomori, M. et al., (2007), Proc. 30th ICRC, Mérida, Mexico

[11] Super-Kamiokande Collaboration, Guillian, G. et al., (2007), Phys. Rev. D 75, 062003

[12] Milagro Collaboration, Abdo, A.A. et al., (2008), Phys. Rev. Lett. 101, 221101

[13] Milagro Collaboration, Abdo, A.A. et al., (2009), Astrophys. J. 698, 2121

[14] EAS-TOP Collaboration, Aglietta, M. et al., (2009), Astrophys. J. 692, L130

[15] ARGO-YBJ Collaboration, Zhang, J.L., (2009), Proc. 31st ICRC, Łódź, Poland

[16] Munakata, K. et al. (2010), Astrophys. J. 712, 1100

[17] Tibet AS $\gamma$ Collaboration, Amenomori, M. et al., (2011), Proc. 32nd ICRC, Beijing China

[18] MINOS Collaboration, de Jong, J. et al., (2011), Proc. 32nd ICRC, Beijing, China

[19] ARGO-YBJ Collaboration, Shuwang, C., et. al. (2011), Proc. 32nd ICRC, Beijing China

[20] ARGO-YBJ Collaboration, Bartoli, B., et al., (2013) Phys. Rev. D 88-8, 082001

[21] HAWC Collaboration, Abeysekara, A.U. et al., (2014), Astrophys. J. 796, 108

[22] ARGO-YBJ Collaboration, Bartoli, B., et al., (2015), Astrophys. J. 809, 90

[23] Tibet AS $\gamma$ Collaboration, Amenomori, M. et al., (2017), Astrophys. J. 836, 153

[24] ARGO-YBJ Collaboration, Bartoli, B., et al., (2018), Astrophys. J., 861, 93

[25] HAWC Collaboration, Abeysekara, A.U. et al. (2018), Astrophys. J., 865, 57

[26] IceCube Collaboration, Abbasi, R. et al., (2010) Astrophys. J. 718, L194 
[27] IceCube Collaboration, Abbasi, R. et al., (2011), Astrophys. J. 74016

[28] IceCube Collaboration, Abbasi et al., (2012), Astrophys. J. 746, 33

[29] IceCube Collaboration, Aartsen, M. et al., (2013), Astrophys. J. 765, 55

[30] IceCube Collaboration, Aartsen, M. et al., (2016), Astrophys. J. 826, 220

[31] IceCube Collaboration, Aartsen M., et al., (2017), PoS(ICRC2017)474

[32] HAWC \& IceCube Collaboration, (2019), Astrophys. J., 871, 96

[33] Fermi-LAT Collaboration, Ajello, M., et al., (2019), arXiv:1903.02905

[34] Giacinti, G. \& Kirk, J.G., (2017), Astrophys. J. 835, 258

[35] Giacinti, G., \& Sigl, G. (2012) Phys. Rev. Lett. 109, 071101

[36] López-Barquero, V., et al. (2017), Astrophys. J., 842, 54

[37] Di Sciascio, G. \& Iuppa, R., (2016), Nucl. and Part. Phys. Proc., 279, 166

[38] Albert A., et al., (2019) arXiv:1902.08429

[39] Abreu P., et al., (2019) Astro2020 APC White Paper, arXiv:1907.07737

[40] IceCube Collaboration, Aartsen, M., et al., (2021), J. Phys. G 6, 060501

[41] Gaisser, T.,(2012), Astropart. Phys., 35, 12

[42] Gaisser, T., et al.,(2013), Front. Phys. (Beijing), 8, 748-758

[43] Li, T.-P., \& Ma, Y.-Q., (1983), Astrophys. J., 272, 317

[44] Whitehorn, N., et al., (2013), Comput. Phys. Commun., 184, 2214-2220 


\section{Full Author List: IceCube Collaboration}

R. Abbasi ${ }^{17}$, M. Ackermann ${ }^{59}$, J. Adams ${ }^{18}$, J. A. Aguilar ${ }^{12}$, M. Ahlers ${ }^{22}$, M. Ahrens ${ }^{50}$, C. Alispach ${ }^{28}$, A. A. Alves Jr. ${ }^{31}$, N. M. $\mathrm{Amin}^{42}$, R. An ${ }^{14}$, K. Andeen ${ }^{40}$, T. Anderson ${ }^{56}$, G. Anton ${ }^{26}$, C. Argüelles ${ }^{14}$, Y. Ashida ${ }^{38}$, S. Axani ${ }^{15}$, X. Bai ${ }^{46}$, A. Balagopal V. ${ }^{38}$, A. Barbano ${ }^{28}$, S. W. Barwick ${ }^{30}$, B. Bastian ${ }^{59}$, V. Basu ${ }^{38}$, S. Baur ${ }^{12}$, R. Bay ${ }^{8}$, J. J. Beatty ${ }^{20,21}$, K.-H. Becker ${ }^{58}$, J. Becker Tjus ${ }^{11}$, C. Bellenghi $^{27}$, S. BenZvi ${ }^{48}$, D. Berley ${ }^{19}$, E. Bernardini ${ }^{59,60}$, D. Z. Besson ${ }^{34,61}$, G. Binder ${ }^{8,9}$, D. Bindig ${ }^{58}$, E. Blaufuss ${ }^{19}$, S. Blot ${ }^{59}$, M. Boddenberg ${ }^{1}$, F. Bontempo ${ }^{31}$, J. Borowka ${ }^{1}$, S. Böser ${ }^{39}$, O. Botner ${ }^{57}$, J. Böttcher ${ }^{1}$, E. Bourbeau ${ }^{22}$, F. Bradascio ${ }^{59}$, J. Braun ${ }^{38}$, S. Bron $^{28}$, J. Brostean-Kaiser ${ }^{59}$, S. Browne ${ }^{32}$, A. Burgman ${ }^{57}$, R. T. Burley ${ }^{2}$, R. S. Busse ${ }^{41}$, M. A. Campana ${ }^{45}$, E. G. Carnie-Bronca ${ }^{2}$, C. Chen ${ }^{6}$, D. Chirkin ${ }^{38}$, K. Choi ${ }^{52}$, B. A. Clark ${ }^{24}$, K. Clark ${ }^{33}$, L. Classen ${ }^{41}$, A. Colemann ${ }^{42}$, G. H. Collin ${ }^{15}$, J. M. Conrad ${ }^{15}$, P. Coppin $^{13}$, P. Correa ${ }^{13}$, D. F. Cowen ${ }^{55,56}$, R. Cross $^{48}$, C. Dappen ${ }^{1}$, P. Dave ${ }^{6}$, C. De Clercq ${ }^{13}$, J. J. DeLaunay ${ }^{56}$, H. Dembinski ${ }^{42}$, K. Deoskar $^{50}$, S. De Ridder ${ }^{29}$, A. Desai ${ }^{38}$, P. Desiati ${ }^{38}$, K. D. de Vries ${ }^{13}$, G. de Wasseige ${ }^{13}$, M. de With ${ }^{10}$, T. DeYoung ${ }^{24}$, S. Dharani ${ }^{1}$, A. Diaz ${ }^{15}$, J. C. Díaz-Vélez ${ }^{38}$, M. Dittmer ${ }^{41}$, H. Dujmovic ${ }^{31}$, M. Dunkman ${ }^{56}$, M. A. DuVernois ${ }^{38}$, E. Dvorak ${ }^{46}$, T. Ehrhardt ${ }^{39}$, P. Eller $^{27}$, R. Engel ${ }^{31,32}$, H. Erpenbeck ${ }^{1}$, J. Evans ${ }^{19}$, P. A. Evenson ${ }^{42}$, K. L. Fan ${ }^{19}$, A. R. Fazely ${ }^{7}$, S. Fiedlschuster ${ }^{26}$, A. T. Fienberg ${ }^{56}$, K. Filimonov ${ }^{8}$, C. Finley ${ }^{50}$, L. Fischer ${ }^{59}$, D. Fox ${ }^{55}$, A. Franckowiak ${ }^{11,59}$, E. Friedman ${ }^{19}$, A. Fritz ${ }^{39}$, P. Fürst ${ }^{1}$, T. K. Gaisser ${ }^{42}$, J. Gallagher $^{37}$, E. Ganster ${ }^{1}$, A. Garcia ${ }^{14}$, S. Garrappa ${ }^{59}$, L. Gerhardt ${ }^{9}$, A. Ghadimi ${ }^{54}$, C. Glaser ${ }^{57}$, T. Glauch ${ }^{27}$, T. Glüsenkamp ${ }^{26}$, A. Goldschmidt $^{9}$, J. G. Gonzalez ${ }^{42}$, S. Goswami ${ }^{54}$, D. Grant ${ }^{24}$, T. Grégoire ${ }^{56}$, S. Griswold ${ }^{48}$, M. Gündüz ${ }^{11}$, C. Günther ${ }^{1}$, C. Haack ${ }^{27}$,

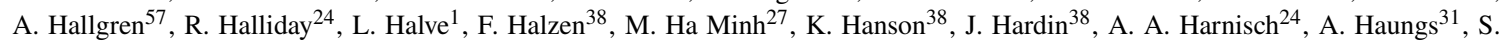
Hauser $^{1}$, D. Hebecker ${ }^{10}$, K. Helbing ${ }^{58}$, F. Henningsen ${ }^{27}$, E. C. Hettinger ${ }^{24}$, S. Hickford ${ }^{58}$, J. Hignight ${ }^{25}$, C. Hill ${ }^{16}$, G. C. Hill ${ }^{2}$, K. D.

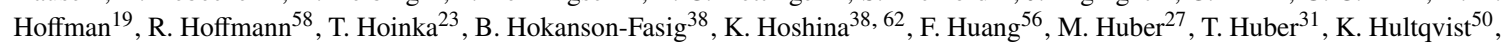
M. Hünnefeld ${ }^{23}$, R. Hussain ${ }^{38}$, S. In ${ }^{52}$, N. Iovine ${ }^{12}$, A. Ishihara ${ }^{16}$, M. Jansson ${ }^{50}$, G. S. Japaridze ${ }^{5}$, M. Jeong ${ }^{52}$, B. J. P. Jones ${ }^{4}$, D. Kang ${ }^{31}$, W. Kang ${ }^{52}$, X. Kang ${ }^{45}$, A. Kappes ${ }^{41}$, D. Kappesser ${ }^{39}$, T. Karg ${ }^{59}$, M. Karl ${ }^{27}$, A. Karle ${ }^{38}$, U. Katz ${ }^{26}$, M. Kauer ${ }^{38}$, M. Kellermann ${ }^{1}$, J. L. Kelley $^{38}$, A. Kheirandish ${ }^{56}$, K. Kin ${ }^{16}$, T. Kintscher ${ }^{59}$, J. Kiryluk ${ }^{51}$, S. R. Klein ${ }^{8,9}$, R. Koirala ${ }^{42}$, H. Kolanoski ${ }^{10}$, T. Kontrimas ${ }^{27}$, L. Köpke $^{39}$, C. Kopper ${ }^{24}$, S. Kopper ${ }^{54}$, D. J. Koskinen ${ }^{22}$, P. Koundal ${ }^{31}$, M. Kovacevich ${ }^{45}$, M. Kowalski ${ }^{10,59}$, T. Kozynets ${ }^{22}$, E. Kun ${ }^{11}$, N. Kurahashi ${ }^{45}$, N. Lad ${ }^{59}$, C. Lagunas Gualda ${ }^{59}$, J. L. Lanfranchi ${ }^{56}$, M. J. Larson ${ }^{19}$, F. Lauber ${ }^{58}$, J. P. Lazar ${ }^{14,38}$, J. W. Lee ${ }^{52}$, K. Leonard $^{38}$, A. Leszczyńska ${ }^{32}$, Y. Li ${ }^{56}$, M. Lincetto ${ }^{11}$, Q. R. Liu ${ }^{38}$, M. Liubarska ${ }^{25}$, E. Lohfink ${ }^{39}$, C. J. Lozano Mariscal ${ }^{41}$, L. Lu ${ }^{38}$,

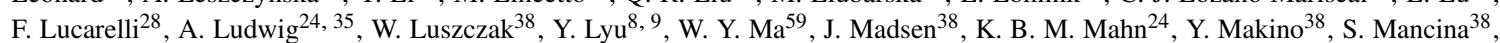

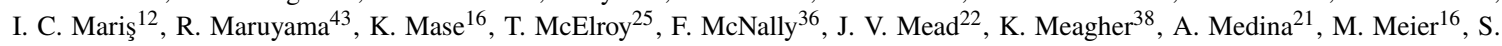
Meighen-Berger $^{27}$, J. Micallef ${ }^{24}$, D. Mockler ${ }^{12}$, T. Montaruli ${ }^{28}$, R. W. Moore ${ }^{25}$, R. Morse ${ }^{38}$, M. Moulai ${ }^{15}$, R. Naab ${ }^{59}$, R. Nagai ${ }^{16}$, U. Naumann $^{58}$, J. Necker ${ }^{59}$, L. V. Nguyễn ${ }^{24}$, H. Niederhausen ${ }^{27}$, M. U. Nisa ${ }^{24}$, S. C. Nowicki ${ }^{24}$, D. R. Nygren ${ }^{9}$, A. Obertacke Pollmann ${ }^{58}$, M. Oehler ${ }^{31}$, A. Olivas ${ }^{19}$, E. O'Sullivan ${ }^{57}$, H. Pandya ${ }^{42}$, D. V. Pankova ${ }^{56}$, N. Park ${ }^{33}$, G. K. Parker ${ }^{4}$, E. N. Paudel ${ }^{42}$, L. Paul ${ }^{40}$, C. Pérez de los $\operatorname{Heros}^{57}$, L. Peters ${ }^{1}$, J. Peterson ${ }^{38}$, S. Philippen ${ }^{1}$, D. Pieloth ${ }^{23}$, S. Pieper ${ }^{58}$, M. Pittermann ${ }^{32}$, A. Pizzuto ${ }^{38}$, M. Plum ${ }^{40}$, Y. Popovych $^{39}$, A. Porcelli ${ }^{29}$, M. Prado Rodriguez ${ }^{38}$, P. B. Price ${ }^{8}$, B. Pries ${ }^{24}$, G. T. Przybylski ${ }^{9}$, C. Raab ${ }^{12}$, A. Raissi ${ }^{18}$, M. Rameez ${ }^{22}$, K. $^{2}$. Rawlins $^{3}$, I. C. Rea ${ }^{27}$, A. Rehman ${ }^{42}$, P. Reichherzer ${ }^{11}$, R. Reimann ${ }^{1}$, G. Renzi ${ }^{12}$, E. Resconi ${ }^{27}$, S. Reusch ${ }^{59}$, W. Rhode ${ }^{23}$, M. Richman ${ }^{45}$, B. Riedel ${ }^{38}$, E. J. Roberts ${ }^{2}$, S. Robertson ${ }^{8,9}$, G. Roellinghoff ${ }^{52}$, M. Rongen ${ }^{39}$, C. Rott ${ }^{49}, 52$, T. Ruhe ${ }^{23}$, D. Ryckbosch ${ }^{29}$, D. Rysewyk $\mathrm{Cantu}^{24}$, I. Safa ${ }^{14,38}$, J. Saffer ${ }^{32}$, S. E. Sanchez Herrera ${ }^{24}$, A. Sandrock ${ }^{23}$, J. Sandroos ${ }^{39}$, M. Santander ${ }^{54}$, S. Sarkar ${ }^{44}$, S. Sarkar ${ }^{25}$, K. Satalecka $^{59}$, M. Scharf ${ }^{1}$, M. Schaufel ${ }^{1}$, H. Schieler ${ }^{31}$, S. Schindler ${ }^{26}$, P. Schlunder ${ }^{23}$, T. Schmidt ${ }^{19}$, A. Schneider ${ }^{38}$, J. Schneider ${ }^{26}$, F. G. Schröder ${ }^{31,42}$, L. Schumacher ${ }^{27}$, G. Schwefer ${ }^{1}$, S. Sclafani ${ }^{45}$, D. Seckel ${ }^{42}$, S. Seunarine ${ }^{47}$, A. Sharma ${ }^{57}$, S. Shefali ${ }^{32}$, M. Silva $^{38}$, B. Skrzypek ${ }^{14}$, B. Smithers ${ }^{4}$, R. Snihur ${ }^{38}$, J. Soedingrekso ${ }^{23}$, D. Soldin ${ }^{42}$, C. Spannfellner ${ }^{27}$, G. M. Spiczak ${ }^{47}$, C. Spiering ${ }^{59,}$, 61 , J. Stachurska $^{59}$, M. Stamatikos ${ }^{21}$, T. Stanev ${ }^{42}$, R. Stein ${ }^{59}$, J. Stettner ${ }^{1}$, A. Steuer ${ }^{39}$, T. Stezelberger ${ }^{9}$, T. Stürwald ${ }^{58}$, T. Stuttard ${ }^{22}$, G. W. Sullivan $^{19}$, I. Taboada ${ }^{6}$, F. Tenholt ${ }^{11}$, S. Ter-Antonyan ${ }^{7}$, S. Tilav ${ }^{42}$, F. Tischbein ${ }^{1}$, K. Tollefson $^{24}$, L. Tomankova ${ }^{11}$, C. Tönnis ${ }^{53}$, S.

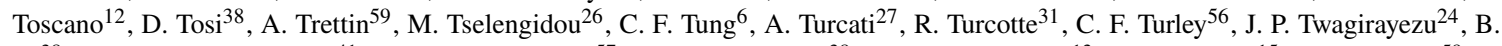
$\mathrm{Ty}^{38}$, M. A. Unland Elorrieta ${ }^{41}$, N. Valtonen-Mattila ${ }^{57}$, J. Vandenbroucke ${ }^{38}$, N. van Eijndhoven ${ }^{13}$, D. Vannerom ${ }^{15}$, J. van Santen $^{59}, \mathrm{~S}$. Verpoest $^{29}$, M. Vraeghe ${ }^{29}$, C. Walck ${ }^{50}$, T. B. Watson ${ }^{4}$, C. Weaver ${ }^{24}$, P. Weigel ${ }^{15}$, A. Weindl ${ }^{31}$, M. J. Weiss ${ }^{56}$, J. Weldert ${ }^{39}$, C. Wendt $^{38}$, J. Werthebach ${ }^{23}$, M. Weyrauch ${ }^{32}$, N. Whitehorn ${ }^{24,35}$, C. H. Wiebusch ${ }^{1}$, D. R. Williams ${ }^{54}$, M. Wolf ${ }^{27}$, K. Woschnagg ${ }^{8}$, G. Wrede ${ }^{26}$, J. Wulff ${ }^{11}$, X. W. Xu $u^{7}$, Y. Xu ${ }^{51}$, J. P. Yanez ${ }^{25}$, S. Yoshida ${ }^{16}$, S. Yu ${ }^{24}$, T. Yuan ${ }^{38}$, Z. Zhang ${ }^{51}$

${ }^{1}$ III. Physikalisches Institut, RWTH Aachen University, D-52056 Aachen, Germany

2 Department of Physics, University of Adelaide, Adelaide, 5005, Australia

${ }^{3}$ Dept. of Physics and Astronomy, University of Alaska Anchorage, 3211 Providence Dr., Anchorage, AK 99508, USA

${ }^{4}$ Dept. of Physics, University of Texas at Arlington, 502 Yates St., Science Hall Rm 108, Box 19059, Arlington, TX 76019, USA

${ }^{5}$ CTSPS, Clark-Atlanta University, Atlanta, GA 30314, USA

${ }^{6}$ School of Physics and Center for Relativistic Astrophysics, Georgia Institute of Technology, Atlanta, GA 30332, USA

${ }^{7}$ Dept. of Physics, Southern University, Baton Rouge, LA 70813, USA

${ }^{8}$ Dept. of Physics, University of California, Berkeley, CA 94720, USA

${ }^{9}$ Lawrence Berkeley National Laboratory, Berkeley, CA 94720, USA

${ }^{10}$ Institut für Physik, Humboldt-Universität zu Berlin, D-12489 Berlin, Germany

${ }^{11}$ Fakultät für Physik \& Astronomie, Ruhr-Universität Bochum, D-44780 Bochum, Germany

12 Université Libre de Bruxelles, Science Faculty CP230, B-1050 Brussels, Belgium

13 Vrije Universiteit Brussel (VUB), Dienst ELEM, B-1050 Brussels, Belgium

14 Department of Physics and Laboratory for Particle Physics and Cosmology, Harvard University, Cambridge, MA 02138, USA

15 Dept. of Physics, Massachusetts Institute of Technology, Cambridge, MA 02139, USA 
${ }^{16}$ Dept. of Physics and Institute for Global Prominent Research, Chiba University, Chiba 263-8522, Japan

${ }^{17}$ Department of Physics, Loyola University Chicago, Chicago, IL 60660, USA

${ }^{18}$ Dept. of Physics and Astronomy, University of Canterbury, Private Bag 4800, Christchurch, New Zealand

${ }^{19}$ Dept. of Physics, University of Maryland, College Park, MD 20742, USA

${ }^{20}$ Dept. of Astronomy, Ohio State University, Columbus, OH 43210, USA

${ }^{21}$ Dept. of Physics and Center for Cosmology and Astro-Particle Physics, Ohio State University, Columbus, OH 43210, USA

${ }^{22}$ Niels Bohr Institute, University of Copenhagen, DK-2100 Copenhagen, Denmark

${ }^{23}$ Dept. of Physics, TU Dortmund University, D-44221 Dortmund, Germany

${ }^{24}$ Dept. of Physics and Astronomy, Michigan State University, East Lansing, MI 48824, USA

${ }^{25}$ Dept. of Physics, University of Alberta, Edmonton, Alberta, Canada T6G 2E1

${ }^{26}$ Erlangen Centre for Astroparticle Physics, Friedrich-Alexander-Universität Erlangen-Nürnberg, D-91058 Erlangen, Germany

${ }^{27}$ Physik-department, Technische Universität München, D-85748 Garching, Germany

${ }^{28}$ Département de physique nucléaire et corpusculaire, Université de Genève, CH-1211 Genève, Switzerland

${ }^{29}$ Dept. of Physics and Astronomy, University of Gent, B-9000 Gent, Belgium

${ }^{30}$ Dept. of Physics and Astronomy, University of California, Irvine, CA 92697, USA

${ }^{31}$ Karlsruhe Institute of Technology, Institute for Astroparticle Physics, D-76021 Karlsruhe, Germany

${ }^{32}$ Karlsruhe Institute of Technology, Institute of Experimental Particle Physics, D-76021 Karlsruhe, Germany

${ }^{33}$ Dept. of Physics, Engineering Physics, and Astronomy, Queen's University, Kingston, ON K7L 3N6, Canada

${ }^{34}$ Dept. of Physics and Astronomy, University of Kansas, Lawrence, KS 66045, USA

${ }^{35}$ Department of Physics and Astronomy, UCLA, Los Angeles, CA 90095, USA

${ }^{36}$ Department of Physics, Mercer University, Macon, GA 31207-0001, USA

${ }^{37}$ Dept. of Astronomy, University of Wisconsin-Madison, Madison, WI 53706, USA

${ }^{38}$ Dept. of Physics and Wisconsin IceCube Particle Astrophysics Center, University of Wisconsin-Madison, Madison, WI 53706, USA

${ }^{39}$ Institute of Physics, University of Mainz, Staudinger Weg 7, D-55099 Mainz, Germany

${ }^{40}$ Department of Physics, Marquette University, Milwaukee, WI, 53201, USA

${ }^{41}$ Institut für Kernphysik, Westfälische Wilhelms-Universität Münster, D-48149 Münster, Germany

${ }^{42}$ Bartol Research Institute and Dept. of Physics and Astronomy, University of Delaware, Newark, DE 19716, USA

${ }^{43}$ Dept. of Physics, Yale University, New Haven, CT 06520, USA

44 Dept. of Physics, University of Oxford, Parks Road, Oxford OX1 3PU, UK

${ }^{45}$ Dept. of Physics, Drexel University, 3141 Chestnut Street, Philadelphia, PA 19104, USA

${ }^{46}$ Physics Department, South Dakota School of Mines and Technology, Rapid City, SD 57701, USA

${ }^{47}$ Dept. of Physics, University of Wisconsin, River Falls, WI 54022, USA

${ }^{48}$ Dept. of Physics and Astronomy, University of Rochester, Rochester, NY 14627, USA

${ }^{49}$ Department of Physics and Astronomy, University of Utah, Salt Lake City, UT 84112, USA

${ }^{50}$ Oskar Klein Centre and Dept. of Physics, Stockholm University, SE-10691 Stockholm, Sweden

${ }^{51}$ Dept. of Physics and Astronomy, Stony Brook University, Stony Brook, NY 11794-3800, USA

52 Dept. of Physics, Sungkyunkwan University, Suwon 16419, Korea

${ }^{53}$ Institute of Basic Science, Sungkyunkwan University, Suwon 16419, Korea

${ }^{54}$ Dept. of Physics and Astronomy, University of Alabama, Tuscaloosa, AL 35487, USA

${ }^{55}$ Dept. of Astronomy and Astrophysics, Pennsylvania State University, University Park, PA 16802, USA

${ }^{56}$ Dept. of Physics, Pennsylvania State University, University Park, PA 16802, USA

57 Dept. of Physics and Astronomy, Uppsala University, Box 516, S-75120 Uppsala, Sweden

${ }^{58}$ Dept. of Physics, University of Wuppertal, D-42119 Wuppertal, Germany

${ }^{59}$ DESY, D-15738 Zeuthen, Germany

${ }^{60}$ Università di Padova, I-35131 Padova, Italy

${ }^{61}$ National Research Nuclear University, Moscow Engineering Physics Institute (MEPhI), Moscow 115409, Russia

${ }^{62}$ Earthquake Research Institute, University of Tokyo, Bunkyo, Tokyo 113-0032, Japan

\section{Acknowledgements}

USA - U.S. National Science Foundation-Office of Polar Programs, U.S. National Science Foundation-Physics Division, U.S. National Science Foundation-EPSCoR, Wisconsin Alumni Research Foundation, Center for High Throughput Computing (CHTC) at the University of Wisconsin-Madison, Open Science Grid (OSG), Extreme Science and Engineering Discovery Environment (XSEDE), Frontera computing project at the Texas Advanced Computing Center, U.S. Department of Energy-National Energy Research Scientific Computing Center, Particle astrophysics research computing center at the University of Maryland, Institute for Cyber-Enabled Research at Michigan State University, and Astroparticle physics computational facility at Marquette University; Belgium - Funds for Scientific Research (FRS-FNRS and FWO), FWO Odysseus and Big Science programmes, and Belgian Federal Science Policy Office (Belspo); Germany - Bundesministerium für Bildung und Forschung (BMBF), Deutsche Forschungsgemeinschaft (DFG), Helmholtz Alliance for Astroparticle Physics (HAP), Initiative and Networking Fund of the Helmholtz Association, Deutsches Elektronen Synchrotron (DESY), and High Performance Computing cluster of the RWTH Aachen; Sweden - Swedish Research Council, Swedish Polar Research Secretariat, Swedish National Infrastructure for Computing (SNIC), and Knut and Alice Wallenberg Foundation; Australia - Australian 
Research Council; Canada - Natural Sciences and Engineering Research Council of Canada, Calcul Québec, Compute Ontario, Canada Foundation for Innovation, WestGrid, and Compute Canada; Denmark - Villum Fonden and Carlsberg Foundation; New Zealand Marsden Fund; Japan - Japan Society for Promotion of Science (JSPS) and Institute for Global Prominent Research (IGPR) of Chiba University; Korea - National Research Foundation of Korea (NRF); Switzerland - Swiss National Science Foundation (SNSF); United Kingdom - Department of Physics, University of Oxford. 